\title{
Mycobacterium abscessus pulmonary disease: individual patient data meta-analysis
}

\author{
Nakwon Kwak ${ }^{1}$, Margareth Pretti Dalcolmo², Charles L. Daley ${ }^{3}$, \\ Geoffrey Eather ${ }^{4}$, Regina Gayoso ${ }^{2}$, Naoki Hasegawa ${ }^{5}$, Byung Woo Jhun $\mathbb{1}^{6}$, \\ Won-Jung Koh (1) ${ }^{6}$, Ho Namkoong ${ }^{7}$, Jimyung Park ${ }^{1}$, Rachel Thomson ${ }^{8}$, \\ Jakko van Ingen $\mathbb{1}^{9}$, Sanne M.H. Zweijpfenning ${ }^{10}$ and Jae-Joon Yim ${ }^{1}$
}

@ERSpublications

For Mycobacterium abscessus pulmonary disease in general, imipenem use is associated with improved outcome. For M. abscessus subsp. abscessus, the use of either azithromycin, amikacin or imipenem increases the likelihood of treatment success. http://ow.ly/w24n30nSakf

Cite this article as: Kwak N, Dalcolmo MP, Daley CL, et al. Mycobacterium abscessus pulmonary disease: individual patient data meta-analysis. Eur Respir J 2019; 54: 1801991 [https://doi.org/10.1183/ 13993003.01991-2018].

ABSTRACT Treatment of Mycobacterium abscessus pulmonary disease (MAB-PD), caused by M. abscessus subsp. abscessus, M. abscessus subsp. massiliense or M. abscessus subsp. bolletii, is challenging.

We conducted an individual patient data meta-analysis based on studies reporting treatment outcomes for MAB-PD to clarify treatment outcomes for MAB-PD and the impact of each drug on treatment outcomes. Treatment success was defined as culture conversion for $\geqslant 12$ months while on treatment or sustained culture conversion without relapse until the end of treatment.

Among 14 eligible studies, datasets from eight studies were provided and a total of 303 patients with MAB-PD were included in the analysis. The treatment success rate across all patients with MAB-PD was 45.6\%. The specific treatment success rates were 33.0\% for M. abscessus subsp. abscessus and $56.7 \%$ for M. abscessus subsp. massiliense. For MAB-PD overall, the use of imipenem was associated with treatment success (adjusted odds ratio (aOR) 2.65, 95\% CI 1.36-5.10). For patients with M. abscessus subsp. abscessus, the use of azithromycin (aOR 3.29, 95\% CI 1.26-8.62), parenteral amikacin (aOR 1.44, 95\% CI 1.05-1.99) or imipenem (aOR 7.96, 95\% CI 1.52-41.6) was related to treatment success. For patients with $M$. abscessus subsp. massiliense, the choice among these drugs was not associated with treatment outcomes.

Treatment outcomes for MAB-PD are unsatisfactory. The use of azithromycin, amikacin or imipenem was associated with better outcomes for patients with M. abscessus subsp. abscessus.

This article has supplementary material available from erj.ersjournals.com

Received: Oct 172018 | Accepted after revision: Feb 202019

This study is registered at PROSPERO with identifier number CRD42017070348.

Copyright OERS 2019 


\section{Introduction}

The incidence and prevalence of pulmonary disease caused by nontuberculous mycobacteria (NTM) are increasing globally [1-4]. Mycobacterium abscessus, comprising of three subspecies, i.e. M. abscessus subsp. abscessus, M. abscessus subsp. massiliense and M. abscessus subsp. bolletii, is the second most common NTM causing pulmonary disease, following Mycobacterium avium complex, in East Asia and the USA $[2,5-8]$.

Treatment for M. abscessus pulmonary disease (MAB-PD) is challenging because of the high frequency of mutational and acquired resistance to commonly used antibiotics [9]. Although macrolides are recommended as a cornerstone of chemotherapy [10,11], mutations in the $\mathrm{rrl}$ gene of $M$. abscessus, which encodes $23 \mathrm{~S}$ rRNA, lead to the acquisition of clarithromycin resistance [12, 13]. Moreover, the erm $(41)$ gene, which encodes a ribosomal methylase, confers inducible resistance to macrolide antibiotics [14]. M. abscessus subsp. abscessus and M. abscessus subsp. bolletii typically express a functional erm(41) gene, and hence demonstrate inducible resistance to macrolide antibiotics. Most M. abscessus subsp. massiliense harbours a mutation in the erm(41) gene that renders it nonfunctional, hence $M$. abscessus subsp. massiliense isolates are intrinsically susceptible to clarithromycin $[12,15]$.

For the treatment of MAB-PD, the American Thoracic Society (ATS)/Infectious Disease Society of America (IDSA) recommends multidrug therapy that includes a macrolide and one or more parenteral drugs (amikacin plus cefoxitin or imipenem) [10]. The British Thoracic Society (BTS) guidelines recommend an antibiotic regimen comprised of intravenous amikacin, tigecycline and imipenem with a macrolide for the initial treatment phase, followed by a continuation phase comprised of nebulised amikacin and a macrolide in combination with additional oral antibiotics [11].

However, the effectiveness of these treatment approaches has not yet been precisely determined, because different studies have adopted different definitions of treatment success $[16,17]$. Some researchers defined sputum culture conversion and maintenance of conversion as treatment success [16], while others reported treatment outcomes based on clinical improvement in addition to sputum culture conversion [17]. Furthermore, the effect of individual drugs has not been elucidated.

Recently, two meta-analyses reporting treatment outcomes for MAB-PD were published [18, 19]. According to these analyses, the treatment success rates for M. abscessus subsp. abscessus and M. abscessus subsp. massiliense were $34.0-41.2 \%$ and 54.0-69.8\%, respectively. However, accurate measurement of the outcomes and role of each drug in MAB-PD treatment could not be determined because these analyses were based on aggregated data provided in published articles.

In this study, we performed a meta-analysis based on individual patient data to clarify treatment outcomes of MAB-PD as well as the impact of each drug on these outcomes.

\section{Methods}

This study was performed in accordance with the PRISMA individual participant data statement [20]. The study protocol was registered with the PROSPERO database (identifier CRD42017070348). Exemption from ethical approval was confirmed by the Institutional Review Board of Seoul National University Hospital (Seoul, South Korea) (1707-007-864).

\section{Search strategy and selection criteria}

We conducted a literature search of the MEDLINE, Embase and Cochrane databases using Medical Subject Heading (MeSH) terms and text words associated with MAB-PD and its treatment. The search query was [(Mycobacterium abscessus) OR (Mycobacterium massiliense) OR (Mycobacterium bolletii)]

Affiliations: ${ }^{1}$ Division of Pulmonary and Critical Care Medicine, Dept of Internal Medicine, Seoul National University College of Medicine, Seoul, South Korea. ${ }^{2}$ Centro de Referencia Helio Fraga, Escola Nacional de Saúde Pública, FIOCRUZ, Rio de Janeiro, Brazil. ${ }^{3}$ Division of Mycobacterial and Respiratory Infections, Dept of Medicine, National Jewish Health, Denver, CO, USA. ${ }^{4}$ Metro South Clinical Tuberculosis Service and Dept of Respiratory and Sleep Medicine, Princess Alexandra Hospital, Brisbane, Australia. ${ }^{5}$ Center for Infectious Diseases and Infection Control, Keio University School of Medicine, Tokyo, Japan. ${ }^{6}$ Division of Pulmonary and Critical Care Medicine, Dept of Medicine, Samsung Medical Center, Sungkyunkwan University School of Medicine, Seoul, South Korea. ${ }^{7}$ Division of Pulmonary Medicine, Dept of Medicine, Keio University School of Medicine, Tokyo, Japan. ${ }^{8}$ Gallipoli Medical Research Institute, University of Queensland, Brisbane, Australia. ${ }^{9}$ Dept of Medical Microbiology, Radboud University Medical Center, Nijmegen, The Netherlands. ${ }^{10}$ Dept of Pulmonary Diseases, Radboud University Medical Center, Nijmegen, The Netherlands.

Correspondence: Jae-Joon Yim, Division of Pulmonary and Critical Care Medicine, Dept of Internal Medicine, Seoul National University College of Medicine, 101 Daehak-Ro, Jongno-Gu, Seoul 110-744, South Korea. E-mail: yimjj@snu.ac.kr 
AND [(Treat $\left.{ }^{\star}\right)$ OR (Therapy)]. The literature search was restricted to articles published between January 1, 1987 and July 31, 2017. The abstracts were independently reviewed by two investigators (N.K. and J.P.). Randomised controlled studies and observational studies reporting treatment outcomes for MAB-PD were selected for a full-text review. The discrepancies were resolved by reaching a consensus with a third investigator ( J-J.Y.).

We selected all studies of patients who were diagnosed as MAB-PD according to the criteria suggested by the ATS/IDSA or BTS $[10,11]$, who underwent chemotherapy, and for whom microbiological and clinical outcomes were reported. We excluded studies with case reports, with patients $<15$ years old and with insufficient reporting of treatment outcomes. Studies mainly comprising patients refractory to previous chemotherapy or patients with acquired mutational macrolide resistance were also excluded.

\section{Data collection and quality assessment}

The corresponding authors of eligible studies were contacted by e-mail and requested to provide the raw data. The following variables were collected: age, sex, body mass index (BMI), past medical history (previous NTM/tuberculosis (TB) treatment, chronic obstructive pulmonary disease (COPD), asthma, cystic fibrosis (CF), bronchiectasis, malignancy or HIV infection), subspecies identification results, radiographic features (nodular bronchiectatic, fibrocavitary or indeterminate), detailed medical treatment history, duration of parenteral $\operatorname{drug}(\mathrm{s})$ use, duration of total treatment, details of adjunctive surgery and treatment outcomes (microbiological, radiographic and symptomatic). If the reply from these authors could not be obtained, repeated contacts were attempted two times more.

The de-identified data provided by the corresponding authors were reviewed by two investigators. All data were merged and transformed into one common dataset. Methodological quality of the studies was evaluated with the Newcastle-Ottawa Scale [21]. The scale was modified with reference to previous reports that described treatment outcomes of single-arm studies [22, 23].

\section{Definitions}

Treatment success was defined as culture conversion for $\geqslant 12$ months while on treatment or sustained culture conversion without relapse until the end of treatment $[10,11,23,24]$. Culture conversion was defined as three or more consecutive negative mycobacterial cultures of sputum. Symptomatic and radiographic improvements were decided based on evaluations by the treating physician at the completion of treatment.

\section{Statistical analysis}

Descriptive variables were summarised with median, interquartile ranges and proportions. These variables were compared between subspecies using Fisher's exact test and the Wilcoxon rank-sum test.

For the analysis of treatment outcomes, the proportions of patients with treatment success, symptomatic and radiographic improvement were calculated. The $95 \%$ confidence intervals for each proportion were obtained with the DerSimonian-Laird random effects model [25]. $I^{2}$ statistics were used to estimate heterogeneity across the studies [26]. The effect of excluded studies on treatment success rates was measured with meta-regression. The potential source of heterogeneity was also assessed with meta-regression [27]. Potential for publication bias was measured using the funnel plot and the Egger test [28].

As a small number of studies and small sample sizes were expected, the one-stage approach was adopted [29]. We used multilevel mixed effects logistic regression with a random intercepts model, and used the random effect parameter for each study and the fixed effect parameter for each intervention to estimate the adjusted odds ratios (aOR) and 95\% confidence intervals of treatment outcomes. Estimates were adjusted for five covariates: age, sex, BMI, radiographic features and presence of respiratory comorbidity [30,31]. Stata version 14.2 (StataCorp, College Station, TX, USA) was used for all statistical analyses.

\section{Results}

\section{Study selection}

We identified a total of 1600 records with our key word-directed literature search, and the titles and abstracts of 1529 articles remained after the removal of duplicates. Of these, 187 articles were selected for full-text review based on the criteria described in the Methods. Full-text reviews narrowed this number down to 14 and the authors were contacted for study participation (Cohen's $\kappa$ for interrater agreement 0.76). The data could not be obtained from six studies [17, 32-36] owing to inaccessibility of the data from four studies, refusal from one study and absence of a response from the authors of one study. Finally, eight studies were the subject of the final analysis: one from Brazil [37], one from Australia [38], one from the USA [39], one from Japan [40], one from the Netherlands [41] and three from South Korea [42-44] (figure 1). Six [37-41,44] out of the eight were retrospective observational studies, while the other two $[42,43]$ were prospective observational studies (table 1 ). Two studies $[42,43]$ were published by the same 


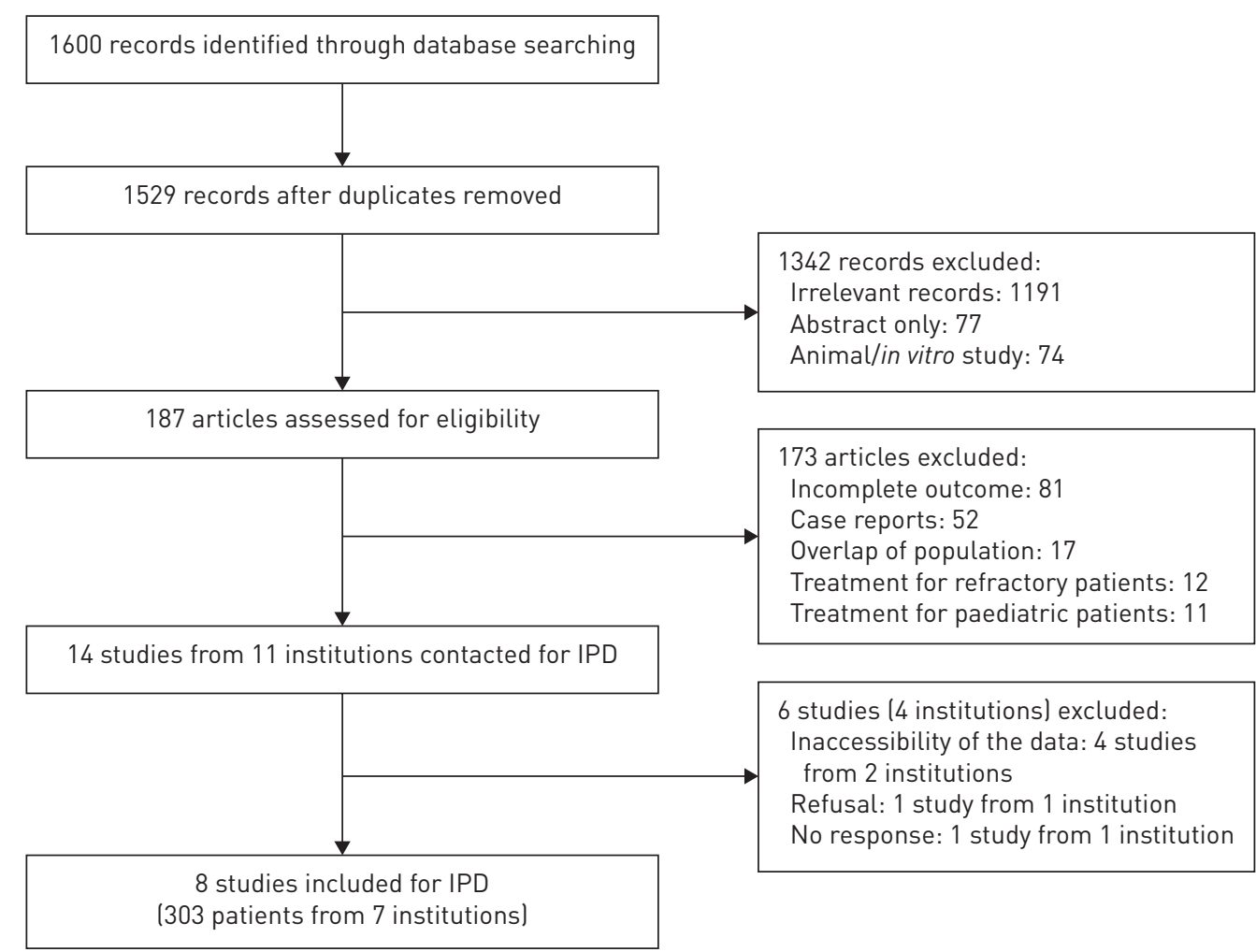

FIGURE 1 PRISMA individual patient data (IPD) flow diagram.

institution and the data from these studies were merged into a combined dataset. Requested and retrieved items from the authors are described in supplementary table E1. The updated data were collected from one study [41]. Four studies [37, 41-43] had a low risk of bias in all aspects, while the others had a risk of bias in terms of representativeness of MAB-PD patients [40], subspecies identification [39] and adequacy of follow-up after treatment $[38,40,44]$ (supplementary table E2). The characteristics of the excluded studies are described in supplementary table E3.

\section{Characteristics of study population}

A total of 303 patients with MAB-PD were included: 126 patients with M. abscessus subsp. abscessus, 95 with $M$. abscessus subsp. massiliense, one with $M$. abscessus subsp. bolletii and 81 without subspecies identification. The subspecies identification was determined based on sequencing of $r p o B$ [38, 40, 42-44], hsp65 [38-43], erm(41) [39, 41], secA [38] and the internal transcribed spacer region [40] or hsp65 PCR restriction enzyme analysis [37].

The median age of the patients was 59 years, $78.6 \%$ were female, 141 (46.5\%) had a previous treatment history for NTM or TB and $12(4.0 \%)$ had CF. Nodular bronchiectatic features were more prevalent among patients with $M$. abscessus subsp. massiliense (74.7\%) than patients with $M$. abscessus subsp.

TABLE 1 Characteristics of the eight studies included in the analysis

\begin{tabular}{|c|c|c|c|c|c|c|}
\hline $\begin{array}{l}\text { First author } \\
\text { [ref.] }\end{array}$ & Study design & $\begin{array}{l}\text { Study } \\
\text { period }\end{array}$ & Region & $\begin{array}{l}\text { Sample } \\
\text { size } n\end{array}$ & $\begin{array}{l}\text { Clinical } \\
\text { setting }\end{array}$ & Type of drug regimen \\
\hline DE MeLLo [37] & Retrospective, cohort & 1993-2011 & Brazil & 26 & Single centre & Individualised \\
\hline ELLender [38] & Retrospective, cohort & $2002-2012$ & Australia & 13 & Single centre & Individualised \\
\hline JARAND [39] & Retrospective, cohort & 2001-2004 & USA & 69 & Single centre & Individualised \\
\hline Кон [42] & Prospective, cohort & $2007-2012$ & South Korea & 71 & Single centre & Standardised and individualised \\
\hline Кон [43] & Prospective, cohort & $2002-2012$ & South Korea & 67 & Single centre & Standardised and individualised \\
\hline Namkoong [40] & Retrospective, cohort & $2004-2013$ & Japan & 13 & Multicentre & Individualised \\
\hline PARK $[44]$ & Retrospective, cohort & $2006-2015$ & South Korea & 36 & Single centre & Individualised \\
\hline VAN INGEN [41] & Retrospective cohort & $1999-2005$ & The Netherlands & 8 & Single centre & Individualised \\
\hline
\end{tabular}


TABLE 2 Baseline characteristics of the 303 patients included in the analysis

\begin{tabular}{|c|c|c|c|c|}
\hline & Total $^{\#}$ & $\begin{array}{l}\text { M. abscessus subsp. } \\
\text { abscessus pulmonary disease }\end{array}$ & $\begin{array}{c}\text { M. abscessus subsp. } \\
\text { massiliense pulmonary disease }\end{array}$ & p-value \\
\hline Patients & 303 & 126 & 95 & \\
\hline Female & $238(78.6)$ & $91(72.2)$ & 79 (83.2) & $0.076^{\S}$ \\
\hline Body mass index $\mathrm{kg} \cdot \mathrm{m}^{-2}$ & $20.5(18.8-22.0)$ & $20.0(18.2-21.9)$ & $20.6(18.8-21.8)$ & $0.385^{+}$ \\
\hline Current or ex-smoker & $60(19.8)$ & $20(15.9)$ & $11(11.6)$ & $0.434^{\S}$ \\
\hline COPD & $20(6.6)$ & $10(7.9)$ & 5 (5.3) & $0.591^{\S}$ \\
\hline Asthma & $9(3.0)$ & $2(1.6)$ & $4(4.2)$ & $0.406^{\S}$ \\
\hline Cystic fibrosis & $12(4.0)$ & 0 & $1(1.1)$ & $0.430^{\S}$ \\
\hline Bronchiectasis & $127(41.9)$ & $63(50.0)$ & $59(62.1)$ & $0.078^{\S}$ \\
\hline Radiographic features prior to treatment & & & & $0.023^{\S}$ \\
\hline Nodular bronchiectatic & $195(64.4)$ & 80 (63.5) & $71(74.7)$ & \\
\hline
\end{tabular}

Data are presented as $\mathrm{n}$, median (interquartile range) or $\mathrm{n}$ (\%), unless otherwise stated. M. abscessus: Mycobacterium abscessus; NTM: nontuberculous mycobacterium; TB: tuberculosis; COPD: chronic obstructive pulmonary disease. Body mass index in 26 patients and smoking history in 13 patients were missing; these values were estimated using multivariate sequential imputation using chained equations. \#: subspecies identifications of $M$. abscessus were missing in 81 patients (one patient was identified as having M. abscessus subsp. bolletii); १: from comparison between patients with $M$. abscessus subsp. abscessus pulmonary disease and M. abscessus subsp. massiliense pulmonary disease; ${ }^{+}$: Wilcoxon rank-sum test; ${ }^{\S}$ : Fisher's exact test.

abscessus (63.5\%; p=0.023) (table 2). Detailed characteristics of the included patients are provided in supplementary table E4.

\section{Treatment outcomes and modalities}

Among the 303 patients with MAB-PD, 164 patients met the criteria for treatment success. The weighted proportion of treatment success for MAB-PD overall was 45.6\% (95\% CI 26.7-64.4\%), while the specific treatment success rates were 33.0\% (95\% CI 16.1-49.8\%) for M. abscessus subsp. abscessus and 56.7\% (95\% CI 9.9-97.8\%) for M. abscessus subsp. massiliense (figure 2). If we excluded studies comprising MAB-PD patients where subspeciation was not performed, the treatment success rates for $M$. abscessus subsp. abscessus and M. abscessus subsp. massiliense pulmonary disease were $27.2 \%$ (95\% CI 10.8-43.5\%) and 57.2\% (95\% CI 10.9-97.5\%), respectively. For M. abscessus subsp. abscessus pulmonary disease, the patients with treatment success received azithromycin $(\mathrm{p}=0.037)$, parenteral amikacin $(\mathrm{p}=0.008)$ or imipenem $(p=0.034)$ more frequently than patients without treatment success, but not cefoxitin $(p=0.444)$. Among patients with MAB-PD as well as patients with M. abscessus subsp. abscessus, durations of total treatment were longer in the treatment failure group than in the success group $(\mathrm{p}<0.001$ and $\mathrm{p}=0.044$, respectively). Duration of parenteral $\operatorname{drug}(\mathrm{s})$ use was also longer in the treatment failure group among patients with MAB-PD $(\mathrm{p}<0.001)$ (table 3$)$.

The weighted proportion of symptomatic improvement after treatment was $64.2 \%$ (95\% CI 51.6-76.7\%) among MAB-PD patients overall: 63.4\% (95\% CI 43.9-81.1\%) for patients with M. abscessus subsp. abscessus and $63.6 \%(95 \%$ CI 15.9-99.6\%) for patients with $M$. abscessus subsp. massiliense (supplementary figure E1). Parenteral amikacin was more frequently prescribed to patients with $M$. abscessus subsp. abscessus or M. abscessus subsp. massiliense who experienced symptomatic improvement ( $\mathrm{p}=0.008$ and $\mathrm{p}=0.001$, respectively) (supplementary table E5).

The weighted proportion of radiographic improvement was $46.8 \%$ (95\% CI 36.8-56.8\%) among MAB-PD patients. Radiographic improvement was attained for 35.7\% (95\% CI 27.2-44.8\%) of patients with $M$. abscessus subsp. abscessus and 70.5\% (95\% CI 33.6-98.0\%) of patients with M. abscessus subsp. massiliense (supplementary figure E2). For M. abscessus subsp. abscessus pulmonary disease, azithromycin rather than clarithromycin was used more commonly in patients with radiographic improvement $(\mathrm{p}=0.006)$ (supplementary table E6).

Treatment outcomes according to age, sex, BMI, respiratory comorbidities and radiographic features are provided in supplementary table E7. 
a)

First author [ref.]

de MeLLo [37]

ELLender [38]

JARAND [39]

KoH $[42,43]$

NAMKoOng [40]

PARK [44]

VAN INGEN [41]

Overall ( $/ 2=90.668 \%, p=0.000)$

0.0

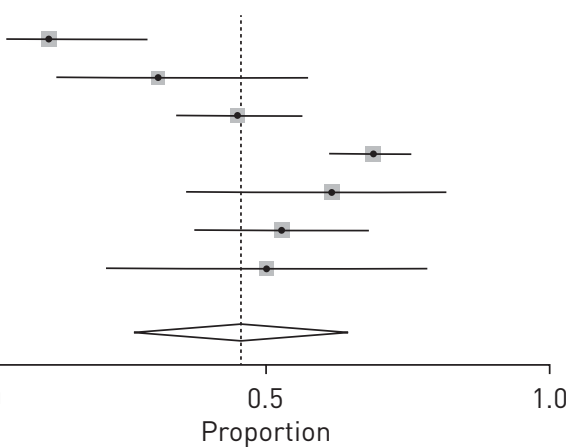

Proportion (95\% CI) Weight \%

$0.115(0.040-0.290) \quad 15.85$

$0.308(0.127-0.576) \quad 13.06$

$0.449(0.338-0.566) \quad 15.95$

$0.688(0.607-0.760) \quad 16.52$

$0.615(0.355-0.823) \quad 12.74$

$0.528(0.370-0.680) \quad 15.08$

$0.500(0.215-0.785) \quad 10.80$

$0.456(0.267-0.644) \quad 100.00$

b)

First author [ref.]

de MeLLo [37]

ELLENDER [38]

$\mathrm{KoH}$ [43]

NAmkoong [40]

PARK [44]

Overall ( $/ 2=73.567 \%, p=0.004)$

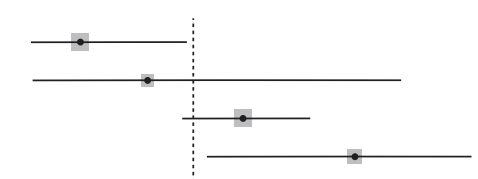

Proportion (95\% Cl) Weight \%

$0.130(0.045-0.321) \quad 25.02$

$0.250(0.046-0.699) \quad 10.33$

$0.418(0.307-0.537) \quad 26.17$

$0.615(0.355-0.823) \quad 17.25$

$0.263(0.118-0.488) \quad 21.22$

$0.330(0.161-0.488) \quad 100.00$

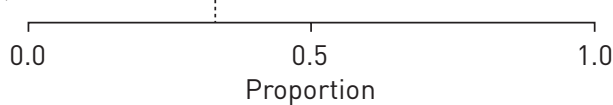

c)

First author [ref.]

dE MeLLo [37]

ELLENDER [38]

JARAND [39]

КоH [42]

PARK [44]

VAN INGEN [41]

Overall $(/ 2=82.588 \%, p=0.000)$

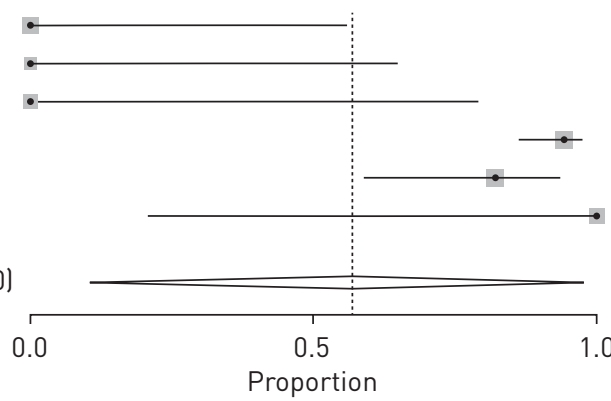

Proportion (95\% Cl) Weight \%

$0.000(0.000-0.561) \quad 16.38$

$0.000(0.000-0.658) \quad 14.43$

$0.000(0.000-0.793) \quad 11.29$

$0.944(0.864-0.978) \quad 24.16$

$0.824(0.590-0.938) \quad 22.46$

$1.000(0.207-1.000) \quad 11.29$

$0.567(0.099-0.978) \quad 100.00$

Proportion

FIGURE 2 Weighted proportion of treatment success for selected studies: a) Mycobacterium abscessus, b) M. abscessus subsp. abscessus and c) M. abscessus subsp. massiliense.

\section{Meta-regression and publication bias}

The concordance of treatment outcomes between included and excluded studies was confirmed with meta-regression (coefficient $-0.04, \mathrm{p}=0.765$ ). The ethnicity of the population (Asian versus non-Asian) (coefficient $0.31, \mathrm{p}=0.052$ ), design of studies (prospective versus retrospective) (coefficient $0.28, \mathrm{p}=0.137$ ) and study quality (low risk of bias versus medium to high risk of bias) (coefficient $-0.02, \mathrm{p}=0.922$ ) did not contribute to the heterogeneity. The funnel plot showed asymmetry (supplementary figure E3), while the Egger test proved no evidence of publication bias $(\mathrm{p}=0.073)$.

\section{Effect of individual drugs on treatment success}

For patients with MAB-PD, the use of imipenem (aOR 2.65, 95\% CI 1.36-5.10) was associated with treatment success, while the other drugs did not show any significant impact on treatment outcomes. For patients with M. abscessus subsp. abscessus specifically, the use of azithromycin (aOR 3.29, 95\% CI 1.268.62), parenteral amikacin (aOR 1.44, 95\% CI 1.05-1.99) or imipenem (aOR 7.96, 95\% CI 1.52-41.6) was associated with higher treatment success, while the use of cefoxitin (aOR 1.22, 95\% CI 0.53-2.86) was not. For patients with M. abscessus subsp. massiliense, the choice among these drugs and treatment outcomes did not show significant correlation (table 4). 
TABLE 3 Comparison of treatment modalities between treatment success and treatment failure groups

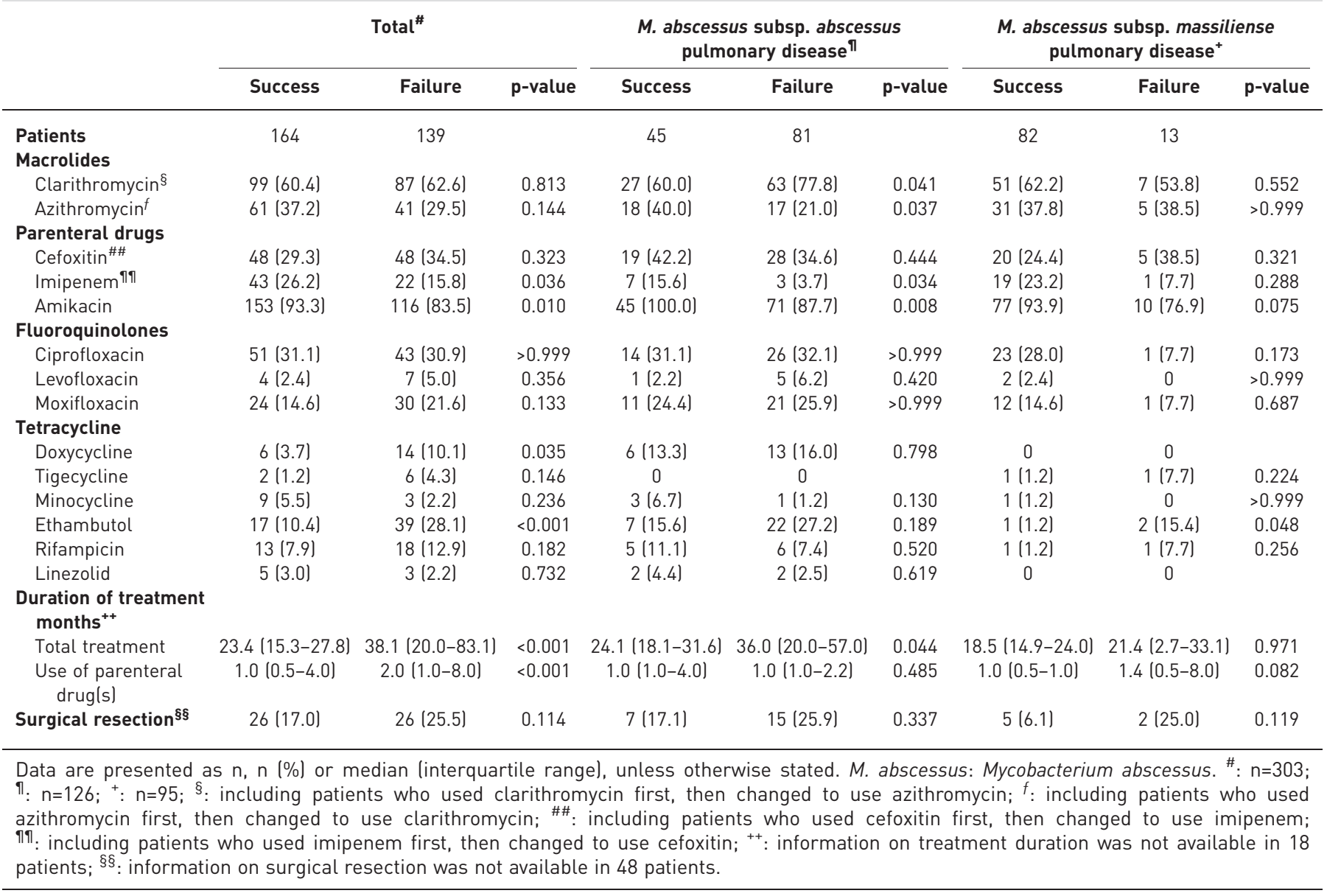

Effect of individual drugs on symptomatic improvement

Among the 303 patients with MAB-PD, parenteral amikacin was associated with symptomatic improvement (aOR 2.95, 95\% CI 1.26-6.91). For patients with M. abscessus subsp. abscessus, the use of azithromycin (aOR 4.58, 95\% CI 1.48-14.2) or amikacin (aOR 19.5, 95\% CI 2.01-189.7) was related to symptomatic improvement, while the use of clarithromycin (aOR 0.20, 95\% CI 0.07-0.62) was not. For

TABLE 4 Association of individual drugs with treatment success

\begin{tabular}{|c|c|c|c|c|c|c|}
\hline & \multicolumn{2}{|l|}{ Total $^{\#}$} & \multicolumn{2}{|c|}{$\begin{array}{l}\text { M. abscessus subsp. } \\
\text { abscessus pulmonary } \\
\text { disease }\end{array}$} & \multicolumn{2}{|c|}{$\begin{array}{c}\text { M. abscessus subsp. } \\
\text { massiliense pulmonary } \\
\text { disease }^{+}\end{array}$} \\
\hline & $\begin{array}{c}\text { Adjusted } \mathrm{OR}^{\S} \\
(95 \% \mathrm{CI})\end{array}$ & $p$-value & $\begin{array}{l}\text { Adjusted OR } \\
(95 \% \mathrm{CI})\end{array}$ & $\overline{p \text {-value }}$ & $\begin{array}{l}\text { Adjusted OR } \\
(95 \% \mathrm{CI})\end{array}$ & $\overline{p \text {-value }}$ \\
\hline Clarithromycin & $0.81(0.47-1.40)$ & 0.438 & $0.33(0.13-0.84)$ & 0.020 & $3.85(0.50-29.6)$ & 0.190 \\
\hline Azithromycin & $1.61(0.93-2.78)$ & 0.085 & $3.29(1.26-8.62)$ & 0.016 & $0.23(0.02-2.42)$ & 0.226 \\
\hline Cefoxitin & $0.61(0.35-1.07)$ & 0.080 & $1.22(0.53-2.86)$ & 0.640 & $0.39(0.04-4.12)$ & 0.429 \\
\hline Imipenem & $2.65(1.36-5.10)$ & 0.005 & $7.96(1.52-41.6)$ & 0.018 & $10.2(0.08-1364.6)$ & 0.353 \\
\hline Amikacin & $2.03(0.74-4.11)$ & 0.181 & $1.44(1.05-1.99)$ & 0.020 & $0.38(0.01-53.1)$ & 0.698 \\
\hline Fluoroquinolone & $0.62(0.36-1.01)$ & 0.076 & $1.24(0.46-3.33)$ & 0.680 & $3.12(0.27-35.9)$ & 0.362 \\
\hline Ethambutol & $0.48(0.23-1.02)$ & 0.060 & $0.54(0.15-1.96)$ & 0.355 & $0.62(0.01-556.3)$ & 0.890 \\
\hline Rifampicin & $0.70(0.29-1.70)$ & 0.425 & $1.21(0.16-9.35)$ & 0.904 & $0.67(0.01-788.6)$ & 0.912 \\
\hline
\end{tabular}


patients with $M$. abscessus subsp. massiliense, amikacin was associated with symptomatic improvement (aOR 31.7, 95\% CI 3.70-271.6) (supplementary table E8).

\section{Effect of individual drugs on radiographic improvement}

For patients with MAB-PD overall, none of the individual drugs was related to radiographic improvement. However, the use of azithromycin (aOR 5.66, 95\% CI 1.86-17.2) rather than clarithromycin (aOR 0.16, 95\% CI 0.06-0.49) was related to the radiographic response for $M$. abscessus subsp. abscessus. Among patients with $M$. abscessus subsp. massiliense, no drugs showed significant correlation to radiographic improvement (supplementary table E9).

\section{Discussion}

We analysed treatment outcomes for MAB-PD and the predictors thereof based on the individual data of 303 patients from seven institutions across six countries. The two main findings of this analysis were: 1) the overall treatment outcomes for MAB-PD, irrespective of subspecies, were unsatisfactory, and 2) the use of azithromycin, amikacin and imipenem was associated with better treatment outcomes among patients with $M$. abscessus subsp. abscessus pulmonary disease. Previous studies have also reported poor treatment outcomes for MAB-PD, especially for M. abscessus subsp. abscessus [18, 19, 45]. According to a recent meta-analysis, the rates of sputum culture conversion were $54 \%$ for MAB-PD altogether, $35 \%$ for M. abscessus subsp. abscessus and 79\% for M. abscessus subsp. massiliense [18]. Our study showed similar findings to these reports: the overall treatment success rate of MAB-PD was $45.6 \%$. Specifically, $33.0 \%$ of patients with $M$. abscessus subsp. abscessus and 56.7\% with M. abscessus subsp. massiliense achieved treatment success. Longer treatment duration in patients with MAB-PD, as well as patients with M. abscessus subsp. abscessus in whom treatments failed, might reflect the difficulties of treatment.

Which macrolide (clarithromycin or azithromycin) is better for the treatment of MAB-PD has not yet been proven and the results of in vitro studies on this issue have been mixed; these mixed results apply both to efficacy and to the differential ability to induce erm(41)-mediated macrolide resistance [38, 39]. One study that was included in the present meta-analysis reported a higher treatment success rate with azithromycin than clarithromycin for patients with MAB-PD [44]. This finding also emerged in our study. The use of azithromycin, rather than clarithromycin, was associated with better outcomes in terms of treatment success as well as the symptomatic and radiographic improvement of patients with M. abscessus subsp. abscessus.

Most clinical isolates of M. abscessus are susceptible to amikacin [46, 47]. In addition, imipenem has the highest in vitro activity among the carbapenems [48] and is preferred over meropenem or ertapenem for the treatment of MAB-PD [10]. In our analysis, the use of amikacin (aOR 1.44, 95\% CI 1.05-1.99) or imipenem (aOR 7.96, 95\% CI 1.52-41.6), but not cefoxitin (aOR 1.22, 95\% CI 0.53-2.86), was associated with treatment success among patients with M. abscessus subsp. abscessus. The importance of the $\beta$-lactam antibiotics is supported by hollow fibre model simulations, which applied cefoxitin because imipenem is too unstable, in which the $\beta$-lactam antibiotic proved to be the main driver of the efficacy of the cefoxitinamikacin-clarithromycin regimen [49]. The lower effectiveness of cefoxitin in clinical practice can be explained in two ways. First, cefoxitin has lower bactericidal and intracellular activity towards M. abscessus subsp. abscessus than imipenem [50]. Second, cefoxitin frequently causes adverse drug events, including leukopenia, thrombocytopenia or drug-induced hepatotoxicity. According to a previous report, 60\% of patients cannot tolerate cefoxitin because of these adverse events [51]. Given the ineffectiveness observed in the current study, frequent adverse events and unavailability of the drug in some regions [11, 33], the use of imipenem rather than cefoxitin for the treatment of MAB-PD may be a reasonable approach.

As it is difficult to achieve long-term sputum culture conversion for MAB-PD, radiographic or symptomatic improvements are suggested as alternative goals of treatment [10]. Quality of life after treatment has also been suggested as a treatment measure [52]. In our study, treatment outcomes in terms of radiographic and symptomatic improvement were included in the analysis. Again, the use of azithromycin rather than clarithromycin was associated with radiographic and symptomatic improvement in $M$. abscessus subsp. abscessus pulmonary disease, although the two macrolides were comparable in M. abscessus subsp. massiliense pulmonary disease.

While azithromycin, amikacin and imipenem were associated with better treatment outcomes in M. abscessus subsp. abscessus pulmonary disease in our study, only amikacin was associated with improvement in symptoms of patients with M. abscessus subsp. massiliense. As most M. abscessus subsp. massiliense has intrinsic susceptibility towards clarithromycin [12], treatment outcomes of patients with M. abscessus subsp. massiliense are better than those with $M$. abscessus subsp. abscessus when using this drug [42, 44]. In our study, treatment success rates for M. abscessus subsp. abscessus and M. abscessus subsp. massiliense pulmonary disease were $27.2 \%$ and $57.2 \%$, respectively, after the exclusion of studies 
including MAB-PD patients without subspeciation. The higher success rate of $M$. abscessus subsp. massiliense pulmonary disease treatment in general may have otherwise masked the superiority of azithromycin over clarithromycin and the effectiveness of imipenem and amikacin.

Our study has several limitations. First, drug susceptibility test results were not available from some institutions and the impact of constitutive clarithromycin resistance could not be adjusted for in the analysis [13]. Second, individual patient data from only eight out of the 14 eligible studies could be obtained. This could limit the generalisability of our results. Third, the asymmetry of the funnel plot and the result of the Egger test suggested the possibility of publication bias, although the Egger test provided a nonsignificant $\mathrm{p}$-value. Fourth, multiple comparisons resulting from the analysis of subspecies and a diverse range of drugs might lead to the risk of type I errors [53]. Fifth, the role of newly adopted drugs, such as tigecycline or the inhaled amikacin, could not be elucidated in our analysis because the numbers of patients using these drugs were too small. Finally, the causality between some drugs and outcomes may not have been fully elucidated because salvage regimens might be associated with poor outcomes regardless of their effectiveness. Despite these limitations, this study has several strengths. This is the first individual patient data meta-analysis of not only patients with MAB-PD but of patients across the whole NTM pulmonary disease spectrum. With the data of individual patients, we were able to evaluate treatment outcomes and the impact of each drug more accurately.

In conclusion, treatment outcomes for MAB-PD are unsatisfactory. For patients with M. abscessus subsp. abscessus, the use of azithromycin, imipenem and amikacin was associated with better treatment outcomes. For patients with M. abscessus subsp. massiliense, the choice among these drugs was not related to treatment outcomes. These findings may prove helpful to clinicians in the design of treatment regimens for patients with MAB-PD.

Author contributions: The authors meet criteria for authorship as recommended by the International Committee of Medical Journal Editors, were fully responsible for all content and were involved at all stages of manuscript development. Study conception and design: N. Kwak and J-J. Yim; data collection: N. Kwak, M.P. Dalcolmo, C.L. Daley, G. Eather, R. Gayoso, N. Hasegawa, B.W. Jhun, W-J. Koh, H. Namkoong, J. Park, J. van Ingen, S.M.H. Zweijpfenning and J-J. Yim; and data interpretation: N. Kwak, M.P. Dalcolmo, C.L. Daley, G. Eather, R. Gayoso, N. Hasegawa, B.W. Jhun, W-J. Koh, H. Namkoong, J. Park, J. van Ingen, S.M.H. Zweijpfenning and J-J. Yim.

Conflict of interest: N. Kwak has nothing to disclose. M.P. Dalcolmo has nothing to disclose. C.L. Daley reports grants and personal fees from Insmed, and personal fees from Horizon, Spero and Johnson and Johnson, outside the submitted work. G. Eather has nothing to disclose. R. Gayoso has nothing to disclose. N. Hasegawa reports grants and personal fees from Insmed Inc., during the conduct of the study; grants from Nikon Corporation, Taisho-Toyama Pharmaceutical Co., Ltd, Eisai Co., Ltd, Daiichi Sankyo Co., Ltd, MSD KK a subsidiary of Merck \& Co. Inc., Sumitomo Dainippon Pharma Co., Ltd, Pfizer Inc., Astellas Pharma Inc., Cepheid Inc., Precision System Science Co., Ltd, and Medical and Biological Laboratories Co., Ltd, outside the submitted work. B.W. Jhun has nothing to disclose. W-J. Koh has received a consultation fee from Insmed Inc. for the Insmed advisory board meeting, not associated with the submitted work. H. Namkoong has nothing to disclose. J. Park has nothing to disclose. R. Thomson reports personal fees for advisory board work from Insmed and Savara, and personal fees for CME talks from Menarini and AstraZeneca, outside the submitted work. J. van Ingen reports personal fees for advisory board membership from Insmed, Spero Therapeutics and Johnson \& Johnson, during the conduct of the study. S.M.H. Zweijpfenning reports personal fees and nonfinancial support from Insmed and Novartis outside the submitted work. J-J. Yim has nothing to disclose.

Support statement: This work was supported by the Seoul National University College of Medicine Research Fund (grant 2320170050). Funding information for this article has been deposited with the Crossref Funder Registry.

\section{References}

1 Brode SK, Marchand-Austin A, Jamieson FB, et al. Pulmonary versus nonpulmonary nontuberculous mycobacteria, Ontario, Canada. Emerg Infect Dis 2017; 23: 1898.

2 Henkle E, Hedberg K, Schafer S, et al. Population-based incidence of pulmonary nontuberculous mycobacterial disease in Oregon 2007 to 2012. Ann Am Thorac Soc 2015; 12: 642-647.

3 Morimoto $\mathrm{K}$, Iwai $\mathrm{K}$, Uchimura $\mathrm{K}$, et al. A steady increase in nontuberculous mycobacteriosis mortality and estimated prevalence in Japan. Ann Am Thorac Soc 2014; 11: 1-8.

4 Namkoong H, Kurashima A, Morimoto K, et al. Epidemiology of pulmonary nontuberculous mycobacterial disease, Japan. Emerg Infect Dis 2016; 22: 1116-1117.

5 Chien JY, Lai CC, Sheng WH, et al. Pulmonary infection and colonization with nontuberculous mycobacteria, Taiwan, 2000-2012. Emerg Infect Dis 2014; 20: 1382.

6 Huang HL, Cheng MH, Lu PL, et al. Epidemiology and predictors of NTM pulmonary infection in Taiwan - a retrospective, five-year multicenter study. Sci Rep 2017; 7: 16300.

7 Lee SK, Lee EJ, Kim SK, et al. Changing epidemiology of nontuberculous mycobacterial lung disease in South Korea. Scand J Infect Dis 2012; 44: 733-738.

8 Prevots DR, Shaw PA, Strickland D, et al. Nontuberculous mycobacterial lung disease prevalence at four integrated health care delivery systems. Am J Respir Crit Care Med 2010; 182: 970-976.

9 Nessar R, Cambau E, Reyrat JM, et al. Mycobacterium abscessus: a new antibiotic nightmare. J Antimicrob Chemother 2012; 67: 810-818. 
10 Griffith DE, Aksamit T, Brown-Elliott BA, et al. An official ATS/IDSA statement: diagnosis, treatment, and prevention of nontuberculous mycobacterial diseases. Am J Respir Crit Care Med 2007; 175: 367-416.

11 Haworth CS, Banks J, Capstick T, et al. British Thoracic Society guidelines for the management of non-tuberculous mycobacterial pulmonary disease (NTM-PD). Thorax 2017; 72: Suppl. 2, iil-ii64.

12 Bastian S, Veziris N, Roux A-L, et al. Assessment of clarithromycin susceptibility in strains belonging to the Mycobacterium abscessus group by erm (41) and rrl sequencing. Antimicrob Agents Chemother 2011; 55: 775-781.

13 Maurer FP, Rüegger V, Ritter C, et al. Acquisition of clarithromycin resistance mutations in the 23S rRNA gene of Mycobacterium abscessus in the presence of inducible erm (41). J Antimicrob Chemother 2012; 67: 2606-2611.

14 Nash KA, Brown-Elliott BA, Wallace RJ. A novel gene, erm (41), confers inducible macrolide resistance to clinical isolates of Mycobacterium abscessus but is absent from Mycobacterium chelonae. Antimicrob Agents Chemother 2009; 53: 1367-1376.

15 Yoshida S, Tsuyuguchi K, Kobayashi T, et al. Discrepancies between the genotypes and phenotypes of clarithromycin-resistant Mycobacterium abscessus complex. Int J Tuberc Lung Dis 2018; 22: 413-418.

16 Koh WJ, Jeon K, Lee NY, et al. Clinical significance of differentiation of Mycobacterium massiliense from Mycobacterium abscessus. Am J Respir Crit Care Med 2011; 183: 405-410.

17 Lyu J, Jang HJ, Song JW, et al. Outcomes in patients with Mycobacterium abscessus pulmonary disease treated with long-term injectable drugs. Respir Med 2011; 105: 781-787.

18 Pasipanodya JG, Ogbonna D, Ferro BE, et al. Systematic review and meta-analyses of the effect of chemotherapy on pulmonary Mycobacterium abscessus outcomes and disease recurrence. Antimicrob Agents Chemother 2017; 61: e01206-17.

19 Diel R, Ringshausen F, Richter E, et al. Microbiological and clinical outcomes of treating non-Mycobacterium avium complex nontuberculous mycobacterial pulmonary disease: a systematic review and meta-analysis. Chest 2017; 152: 120-142.

20 Stewart LA, Clarke M, Rovers M, et al. Preferred reporting items for a systematic review and meta-analysis of individual participant data: the PRISMA-IPD statement. JAMA 2015; 313: 1657-1665.

21 Wells GA, Shea B, O'Connell D, et al. The Newcastle-Ottawa Scale (NOS) for assessing the quality of nonrandomised studies in meta-analyses. 2008. www.ohri.ca/programs/clinical_epidemiology/oxford.asp Date last accessed: February 9, 2019.

22 Dey T, Brigden G, Cox H, et al. Outcomes of clofazimine for the treatment of drug-resistant tuberculosis: a systematic review and meta-analysis. J Antimicrob Chemother 2012; 68: 284-293.

23 Kwak N, Park J, Kim E, et al. Treatment outcomes of Mycobacterium avium complex lung disease: a systematic review and meta-analysis. Clin Infect Dis 2017; 65: 1077-1084.

24 van Ingen J, Aksamit $\mathrm{T}$, Andrejak $\mathrm{C}$, et al. Treatment outcome definitions in nontuberculous mycobacterial pulmonary disease: an NTM-NET consensus statement. Eur Respir J 2018; 51: 1800170

25 DerSimonian R, Laird N. Meta-analysis in clinical trials. Control Clin Trials 1986; 7: 177-188.

26 Higgins JP, Thompson SG, Deeks JJ, et al. Measuring inconsistency in meta-analyses. BMJ 2003; 327: 557.

27 Thompson SG, Higgins JP. How should meta-regression analyses be undertaken and interpreted? Stat Med 2002; 21: 1559-1573.

28 Harbord RM, Egger M, Sterne JA. A modified test for small-study effects in meta-analyses of controlled trials with binary endpoints. Stat Med 2006; 25: 3443-3457.

29 Burke DL, Ensor J, Riley RD. Meta-analysis using individual participant data: one-stage and two-stage approaches, and why they may differ. Stat Med 2017; 36: 855-875.

30 Andréjak C, Thomsen VØ, Johansen IS, et al. Nontuberculous pulmonary mycobacteriosis in Denmark: incidence and prognostic factors. Am J Respir Crit Care Med 2010; 181: 514-521.

31 Hayashi M, Takayanagi N, Kanauchi T, et al. Prognostic factors of 634 HIV-negative patients with Mycobacterium avium complex lung disease. Am J Respir Crit Care Med 2012; 185: 575-583.

32 Jo KU, Park SJ, Hong SC, et al. Long-term outcome of treatment of Mycobacterium abscessus pulmonary disease. Tuberc Respir Dis 2007; 62: 98-104.

33 Harada T, Akiyama Y, Kurashima A, et al. Clinical and microbiological differences between Mycobacterium abscessus and Mycobacterium massiliense lung diseases. J Clin Microbiol 2012; 50: 3556-3561.

34 Lyu J, Kim BJ, Kim BJ, et al. A shorter treatment duration may be sufficient for patients with Mycobacterium massiliense lung disease than with Mycobacterium abscessus lung disease. Respir Med 2014; 108: 1706-1712.

35 Martiniano SL, Wagner BD, Levin A, et al. Safety and effectiveness of clofazimine for primary and refractory nontuberculous mycobacterial infection. Chest 2017; 152: 800-809.

36 Roux A-L, Catherinot E, Soismier N, et al. Comparing Mycobacterium massiliense and Mycobacterium abscessus lung infections in cystic fibrosis patients. J Cyst Fibros 2015; 14: 63-69.

37 de Mello KGC, Mello FCQ, Borga L, et al. Clinical and therapeutic features of pulmonary nontuberculous mycobacterial disease, Rio de Janeiro, Brazil. Emerg Infect Dis 2013; 19: 393.

38 Ellender CM, Law DB, Thomson RM, et al. Safety of IV amikacin in the treatment of pulmonary non-tuberculous mycobacterial disease. Respirology 2016; 21: 357-362.

39 Jarand J, Levin A, Zhang L, et al. Clinical and microbiologic outcomes in patients receiving treatment for Mycobacterium abscessus pulmonary disease. Clin Infect Dis 2011; 52: 565-571.

40 Namkoong H, Morimoto K, Nishimura T, et al. Clinical efficacy and safety of multidrug therapy including thrice weekly intravenous amikacin administration for Mycobacterium abscessus pulmonary disease in outpatient settings: a case series. BMC Infect Dis 2016; 16: 396.

41 van Ingen J, de Zwaan R, Dekhuijzen RP, et al. Clinical relevance of Mycobacterium chelonae-abscessus group isolation in 95 patients. $J$ Infect 2009; 59: 324-331.

42 Koh WJ, Jeong BH, Jeon $\mathrm{K}$, et al. Oral macrolide therapy following short-term combination antibiotic treatment of Mycobacterium massiliense lung disease. Chest 2016; 150: 1211-1221.

43 Koh WJ, Jeong BH, Kim SY, et al. Mycobacterial characteristics and treatment outcomes in Mycobacterium abscessus lung disease. Clin Infect Dis 2017; 64: 309-316.

44 Park J, Cho J, Lee C-H, et al. Progression and treatment outcomes of lung disease caused by Mycobacterium abscessus and Mycobacterium massiliense. Clin Infect Dis 2017; 64: 301-308. 
45 Morimoto K, Nakagawa T, Asami T, et al. Clinico-microbiological analysis of 121 patients with pulmonary Mycobacteroides abscessus complex disease in Japan - an NTM-JRC study with RIT. Respir Med 2018; 145: 14-20.

46 Huang YC, Liu MF, Shen GH, et al. Clinical outcome of Mycobacterium abscessus infection and antimicrobial susceptibility testing. J Microbiol Immunol Infect 2010; 43: 401-406.

47 Park S, Kim S, Park EM, et al. In vitro antimicrobial susceptibility of Mycobacterium abscessus in Korea. J Korean Med Sci 2008; 23: 49-52.

48 Brown-Elliott BA, Killingley J, Vasireddy S, et al. In vitro comparison of ertapenem, meropenem, and imipenem against isolates of rapidly growing mycobacteria and Nocardia using broth microdilution and E-tests. J Clin Microbiol 2016; 54: 00298-16.

49 Ferro BE, Srivastava S, Deshpande D, et al. Failure of the amikacin, cefoxitin, and clarithromycin combination regimen for pulmonary Mycobacterium abscessus. Antimicrob Agents Chemother 2016; 60: 6374-6376

50 Lefebvre A-L, Dubée V, Cortes M, et al. Bactericidal and intracellular activity of $\beta$-lactams against Mycobacterium abscessus. J Antimicrob Chemother 2016; 71: 1556-1563.

51 Jeon K, Kwon OJ, Lee NY, et al. Antibiotic treatment of Mycobacterium abscessus lung disease: a retrospective analysis of 65 patients. Am J Respir Crit Care Med 2009; 180: 896-902.

52 Czaja CA, Levin AR, Cox CW, et al. Improvement in quality of life after therapy for Mycobacterium abscessus group lung infection. A prospective cohort study. Ann Am Thorac Soc 2016; 13: 40-48.

53 Shaffer JP. Multiple hypothesis testing. Annu Rev Psychol 1995; 46: 561-584. 